\title{
Trophoblast-derived hyaluronan promotes the regulatory phenotype of decidual macrophages
}

\author{
Songcun Wang ${ }^{1, *}$, Fengrun Sun ${ }^{1, *}$, Mutian $\mathrm{Han}^{2}$, Yinghua $\mathrm{Liu}^{2}$, Qinyan Zou², Fuxin Wang², \\ Yu Tao ${ }^{1}$, Dajin $\mathrm{Li}^{1}$, Meirong $\mathrm{Du}^{1}$, Hong $\mathrm{Li}^{2}$ and Rui Zhu ${ }^{2}$ \\ ${ }^{1}$ Laboratory for Reproductive Immunology, NHC Key Lab of Reproduction Regulation (Shanghai Institute of Planned \\ Parenthood Research), Shanghai Key Laboratory of Female Reproductive Endocrine Related Diseases, Hospital of \\ Obstetrics and Gynecology, Fudan University Shanghai Medical College, Shanghai, China and ${ }^{2}$ The Affiliated \\ Suzhou Hospital of Nanjing Medical University/Suzhou Municipal Hospital, Suzhou, China
}

Correspondence should be addressed to R Zhu: summerrui2006@aliyun.com or M Du: dmrlq1973@sina.cn or H Li: hongziszivf@163.com

*(S Wang and F Sun contributed equally to this work)

\begin{abstract}
There is delicate crosstalk between fetus-derived trophoblasts (Tros) and maternal cells during normal pregnancy. Dysfunctions in interaction are highly linked to some pregnancy complications, such as recurrent spontaneous abortion (RSA), pre-eclampsia and fetal growth restriction. Hyaluronan (HA), the most abundant component of extracellular matrix, has been reported to act as both a pro- and an anti-inflammatory molecule. Previously, we reported that HA promotes the invasion and proliferation of Tros by activating PI3K/Akt and MAPK/ERK1/2 signaling pathways. While lower HA secretion by Tros was observed during miscarriages than that during normal pregnancies, in the present study, we further confirmed that higher secretion of HA by Tros could induce M2 polarization of macrophages at the maternal-fetal interface by interacting with CD44 and activating the downstream PI3K/AktSTAT-3/STAT-6 signaling pathways. Furthermore, HA could restore the production of IL-10 and other normal pregnancy markers by decidual macrophages (dM $\rho s)$ from RSA. These findings underline the important roles of HA in regulating the function of $\mathrm{dM} \varphi s$ and maintaining a normal pregnancy.

Reproduction (2019) 157 189-198
\end{abstract}

\section{Introduction}

In a successful pregnancy, the maternal immune system undergoes numerous changes in order to avoid the alienation of the semi-allogeneic fetus, while at the same time protecting the woman against pathogens. This balance is mainly established locally at the maternalfetal interface, where the extravillous trophoblast cells invade the decidua and come into contact with the decidual immune cells (DICs). Failure to establish and maintain this proper crosstalk is associated with complications of pregnancy, such as pre-eclampsia and recurrent spontaneous abortion (RSA) (Arck \& Hecher 2013).

Macrophages are the second most abundant leucocytes (10-20\% of the DICs) and the most important specialized antigen-presenting cells at the maternalfetal interface (Svensson-Arvelund \& Ernerudh 2015). Decidual macrophages (dM $\mathrm{ss}$ ) are associated with the success of pregnancy by promoting decidual vascular remodeling (Lash et al. 2016) and clearance of cell debris (Egashira et al. 2017). The depletion of macrophages after conception has been reported to cause embryo implantation arrest (Care et al. 2013). Parallel to the T helper-1 (Th1)/T helper-2 (Th2) paradigm, environmental cues and molecular mediators polarize macrophages as type 1 macrophages (M1) and type 2 macrophages (M2). With the high expression of major histocompatibility complex class II (MHC II), CD80, CD86 and IL-12, M1 are more effective at antigen clearance and switching T-cell responses toward Th1 immune response (Mills et al. 2000). It has been reported that M1 play crucial roles in implantation (Jaiswal et al. 2012). While characterized by M2-type markers (CD163, CD206, CD209 and IL-10), M2 have more immunosuppressive capacities, contributing to tissue remodeling and promoting Th2 immune responses (Murray et al. 2014). It seems that $\mathrm{d} M \varphi \mathrm{s}$ maintain the balance between proinflammatory $\mathrm{M} 1$ and anti-inflammatory $\mathrm{M} 2$ during normal pregnancies. Imbalance of M1/M2 leads to a pathological pregnancy, such as RSA (Shimada et al. 2018, Tsao et al. 2018).

Hyaluronan (HA) is an unbranched polymer composed of repeating glucuronic acid and $\mathrm{N}$-acetyl glucosamine 
disaccharide units, and it is found ubiquitously in the extracellular matrix (ECM) of all mammalian tissues. $\mathrm{HA}$ is protective against infection-mediated preterm birth as it is crucial in maintaining an epithelial and mucosal barrier to limit pathogen infiltration of the lower reproductive tract during pregnancy (Akgul et al. 2014). In addition to its structural role in the ECM, HA has diverse functions in cell growth, migration and adhesion, dependent on the HA size and expression of HA-interacting molecules such as CD44 (Dicker et al. 2014). During early pregnancy, high-molecular-weight $\mathrm{HA}$ (HMW-HA) enhances the proliferation and invasive properties of trophoblasts (Tros) and inhibits apoptosis by activating PI3K/AKT and MAPK/ERK1/2 signaling pathways (Zhu et al. 2013a). Meanwhile low-molecularweight HA (LMW-HA) has been reported to play a role in promoting angiogenesis and inflammation (Jiang et al. 2005).

Emerging evidence has suggested that HA creates a specific microenvironment that is favorable for tumor angiogenesis, invasion, metastasis and immune escape (Chanmee et al. 2016). Recently, it has been revealed in a study that the HA-CD44-ERK1/2-STAT3 pathway serves as an important regulator in the formation of M2-like tumor-associated macrophages (TAMs) (Zhang et al. 2016). Given the pseudo-malignant nature of the placenta, it is possible that HA plays a role in maternalfetal tolerance by regulating the function of $d M \varphi s$. Here we showed that, during normal pregnancies in humans, Tros-derived HA induces the M2 polarization of $\mathrm{dM} \varphi \mathrm{s}$ by interacting with CD44 and activating the PI3K/AktSTAT3/STAT-6 pathway.

\section{Materials and methods}

\section{Human samples}

First-trimester (gestational age: 6-12 weeks) human villous and decidual tissues were obtained from normal pregnancies (terminated for non-medical reasons, $n=33$ ) and clinical miscarriages (two or more miscarriages diagnosed as RSA, excluding genetic, endocrine and anatomic abnormalities, infections, poor health habits and so forth; $n=28$ ). The miscarried decidual tissues were obtained very soon after they were identified. All participants provided written informed consent. The Human Research Ethics Committee of the Obstetrics and Gynecology Hospital of Fudan University approved the collection and use of the samples. All the methods were carried out in accordance with the approved guidelines.

\section{Human cell isolation}

Tros were isolated by trypsin-DNase-I digestion and discontinuous Percoll gradient centrifugation, as previously described (Wang et al. 2016).

DICs were isolated by collagenase IV/DNase-I digestion and discontinuous Percoll gradient centrifugation from the decidual tissue of normal pregnancies and RSA, as previously described (Wang et al. 2015, 2016). dM $\varphi$ s were isolated form DICs by magnetic affinity cell sorting using CD14 microbeads (Miltenyi Biotec).

\section{Treatment of $d M \varphi s$}

Freshly isolated $\mathrm{dM} \varphi \mathrm{s}$ were cultured with $\mathrm{HMW}-\mathrm{HA}$ (50 or $100 \mu \mathrm{g} / \mathrm{mL}$ ) or medium-molecular-weight HA (MMW-HA) $(50$ or $100 \mu \mathrm{g} / \mathrm{mL}$ ) or LMW-HA (50 or $100 \mu \mathrm{g} / \mathrm{mL}$ ) (all from R\&D Systems) with or without the pretreatment of neutralizing anti-CD44 antibody $(20 \mu \mathrm{g} / \mathrm{mL}$, Clone 5F12, Scientific Inc, Fremont, $\mathrm{CA}, \mathrm{USA}$ ) at $37^{\circ} \mathrm{C}$ in $5 \% \mathrm{CO}_{2}$ for $48 \mathrm{~h}$. In some experiments, the cells were pretreated with the PI3K signaling pathway inhibitor LY294002 $(50 \mathrm{mM}$, Cell Signaling Technology) or MEK1/2 inhibitor U0126 (30 mM, Cell Signaling Technology) for 30 min before HA treatment.

Freshly isolated Tros were seeded at a density of $2 \times 10^{5}$ cells/ $\mathrm{mL}$ per well in 24-well Matrigel-coated plates overnight. The cells were washed twice with phosphate-buffered saline and the supernatants were collected after culturing for $48 \mathrm{~h}$. The supernatants were harvested and centrifuged at 2000g, and then moved to a new tube and stored at $-80^{\circ} \mathrm{C}$. dM $\varphi$ s were cultured with the supernatants of Tros (TCM). In some wells, an HA antagonist (pep-1) and control peptides (Cpep, $100 \mathrm{mg} /$ $\mathrm{mL}$, Invitrogen Life Technologies Corp) with or without the pretreatment of neutralizing anti-CD44 $(20 \mu \mathrm{g} / \mathrm{mL})$ antibody were added. The cells were then harvested for analysis by flow cytometry after $48 \mathrm{~h}$ of co-culture.

\section{Western blot analysis}

$\mathrm{dM} \varphi \mathrm{s}$ were stimulated by $100 \mu \mathrm{g} / \mathrm{mL}$ HMW-HA for $0,15,30$, 60 or $120 \mathrm{~min}$. The cells were harvested and homogenized in an ice-cold radioimmunoprecipitation assay (RIPA) lysis buffer. Total cell lysates were collected, and equal quantities of protein were separated by SDS-PAGE and blotted onto PVDF membranes. The PVDF membranes were blocked with Trisbuffered saline (TBS) containing 5\% skimmed milk powder for $1 \mathrm{~h}$ at room temperature and incubated with anti-phosphoAkt (1:1000, Cell Signaling Technology), anti-Akt (1:1000, Cell Signaling Technology) or anti-GAPD H mAb (1:3000, Cell Signaling Technology) overnight at $4{ }^{\circ} \mathrm{C}$. Then, the membranes were washed with $1 \times$ TBS/Tween 20 buffer three times and incubated with horseradish peroxidase-conjugated polyclonal secondary antibodies for $1 \mathrm{~h}$ at room temperature. The membranes were developed with the enhanced plus chemiluminescence assay.

\section{Flow cytometry}

The expression of cell-surface molecules and intracellular cytokines was evaluated using flow cytometry. The following were used: fluorescein isothiocyanate (FITC)-conjugated antihuman CD80, CD209 and CD14, phycoerythrin(PE)-conjugated anti-human CXCL10, CCL22, CCL17, CXCL9, CD80, CD163 and CD86, PE/CY7-conjugated anti-human CD86, CD14, IL-10 and TNF- $\alpha$, allophycocyanin (APC)-conjugated antihuman CD206, and ICOSL, Alexa Fluor 647-conjugated anti-human IL-12, Brilliant Violet 421-conjugated anti-human 
PDL-1, PDL-2, HLA-DR, CD163 and IL-4 and Brilliant Violet 510-conjugated anti-human CD14, PDL-1 (BioLegend, San Diego, CA, USA). For intracellular staining, cells were fixed and permeabilized using the Fix/Perm Kit (BioLegend). A minimum of 10,000 events were acquired using a BeckmanCoulter CyAn ADP flow cytometer and analyzed with Flowjo software (Tree Star, Ashland, OR, USA).

\section{Statistical analysis}

The significance of differences between two groups was determined by Dunnett's post hoc $t$-test. Multiple groups were analyzed by one-way or two-way ANOVA with Bonferroni's posttests using Prism version 5 software (GraphPad). For all statistical tests, $P$ values of $<0.05$ were considered statistically significant.

\section{Results}

\section{Effects of HA on dM $\rho$ s during early pregnancy}

In order to investigate the potential role of $\mathrm{HA}$ in the function of $\mathrm{dM} \varphi \mathrm{s}$ during normal early pregnancies in humans, we first studied the effect of HA with different molecular weights on the expression of $\mathrm{MHC}$ II and co-stimulatory molecules of dM $\varphi$ s. As shown in Fig. 1A, $100 \mu \mathrm{g} / \mathrm{mL}$ HMW-HA (>950 kDa) downregulated the expression of CD80 and CD86 but upregulated that of PDL-1 and PDL-2 on dM $\varphi$ s. PDL-1 and PDL-2 are related to the alternative activated phenotype in macrophages and are mechanisms of T-cell suppression by alternative activated macrophages (Smith et al. 2004, Terrazas et al. 2005). The expression of ICOSL and HLA-DR did not vary with the treatment with HA (Supplementary Fig. 1A, see section on supplementary data given at the end of this article).

Highly expressed pattern recognition receptors such as CD163 and CD206 on dMps play important roles in clearing pathogens at the maternal-fetal interface (Tang et al. 2015). We found an increase of CD163 and CD206 on dM $\varphi s$ after treatment with HMW-HA (Fig. 1B). HMW-HA also increased the production of IL-10 by dM $\varphi$ s (Fig. 1C), but had no effect on the production of IL-12 (Supplementary Fig. 1B). Apart from these surface molecules and cytokines, chemokines have also been used as biomarkers of M1/M2 (Zhang et al. 2017). HMW-HA increased M2-associated CCL17 and CCL22 but decreased M1-associated CXCL10 expression on dM $\varphi s$ (Fig. 1D). Although LMW-HA was reported to induce a classically activated-like state of macrophages (Rayahin et al. 2015), as shown in Fig. 1, we did not observe the effects of MMW-HA (75-350kDa) and LMW-HA (15-40 kDa) on the phenotype of dM $\varphi s$.
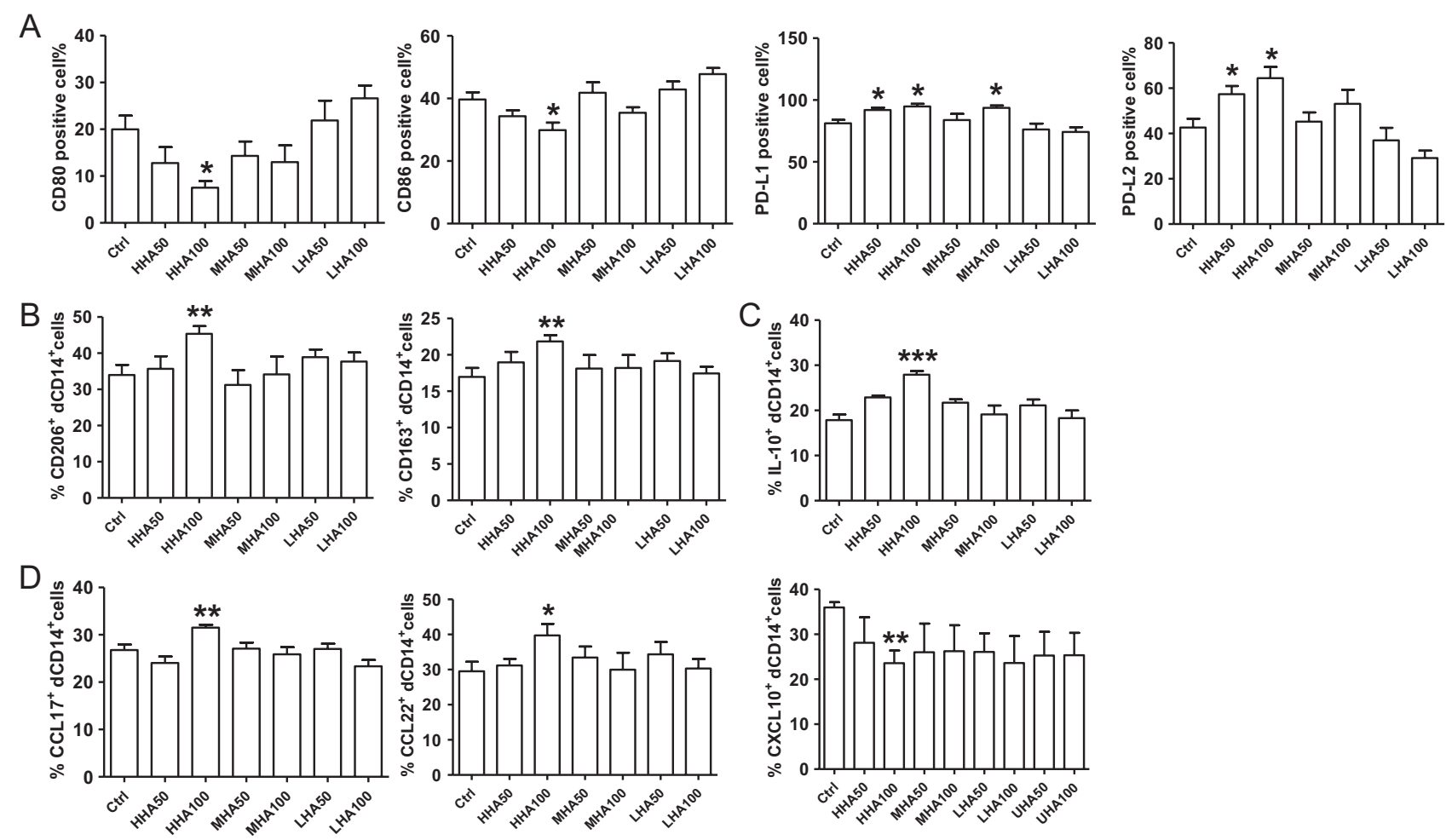

Figure 1 Effects of $\mathrm{HA}$ on $\mathrm{dM} \varphi \mathrm{s}$ during early pregnancy. (A) Quantitation of flow cytometric analysis of the expression of $\mathrm{MHC}$ II and co-stimulatory molecules by dM $\varphi$ s stimulated with HMW-HA (HHA, 50 or $100 \mu \mathrm{g} / \mathrm{mL}$ ) or MMW-HA (MHA, 50 or $100 \mu \mathrm{g} / \mathrm{mL})$ or LMW-HA (LHA, 50 or $100 \mu \mathrm{g} / \mathrm{mL}$ ). (B, C and D) Quantitation of flow cytometric analysis of the expression of CD206, CD163 cytokines and chemokines by dM $\varphi s$ stimulated with the indicated concentrations of HMW-HA, MMW-HA or LMW-HA. Data are represented as the mean \pm standard error of the mean (S.E.M.) for three to five separate experiments. $n=12 \mathrm{dM} \varphi \mathrm{s}$ in the first trimester of normal pregnancies. ${ }^{*} P<0.05,{ }^{* *} P<0.01,{ }^{* * *} P<0.001$. 


\section{HMW-HA induces $\mathrm{M} 2$ polarization through CD44}

CD44 is the principal receptor of $\mathrm{HA}$, and HA-CD44 signaling has long been known to play a role in immune regulation (Heldin et al. 2008). In order to determine whether the functional regulation of $\mathrm{HMW}-\mathrm{HA}$ on $\mathrm{dM} \varphi \mathrm{s}$ is dependent on CD44, we first examined the expression of CD44 on DICs and found that about $80 \%$ of $\mathrm{dM} \varphi s$ expressed CD44 (Fig. 2A and B). Then, we used an anti-CD44 neutralizing antibody to directly analyze the interaction of HMW-HA with CD44 on dMps. Pretreatment with anti-CD44 monoclonal antibodies (mAbs) notably reversed the induction of $\mathrm{M} 2$ polarization by HMW-HA, as confirmed by lower expression of PDL1, PDL-2, CD206, CD209, CD163, IL-10, CCL17 and CCL22, and the higher expression of CD80, CD86 and CXCL10 on dMps (Fig. 2C).

\section{PI3K/Akt-STAT-3/STAT-6 pathways are involved in M2 polarization}

We previously reported that HMW-HA could activate PI3K/Akt and MAPK/ERK1/2 signaling pathways in Tros and decidual stromal cells during early pregnancies (Zhu et al. 2013a,b). In order to further understand the intracellular mechanism after the binding of HMW-HA to CD44 on $\mathrm{dM} \varphi \mathrm{s}$, the effect of the specific signal transduction inhibitors on $\mathrm{dM} \varphi \mathrm{s}$ was examined. The results showed that U0126 (an inhibitor of ERK1/2) had no effect on HMW-HA-mediated M2 polarization. Conversely, LY294002 (an inhibitor of PI3K) markedly reversed this effect (Fig. 3A). Additionally, treatment with U0126 or LY294002 alone did not affect the expression of M1/M2-associated markers on dM $\mathrm{d}$, suggesting that the reversal effect of LY294002 was
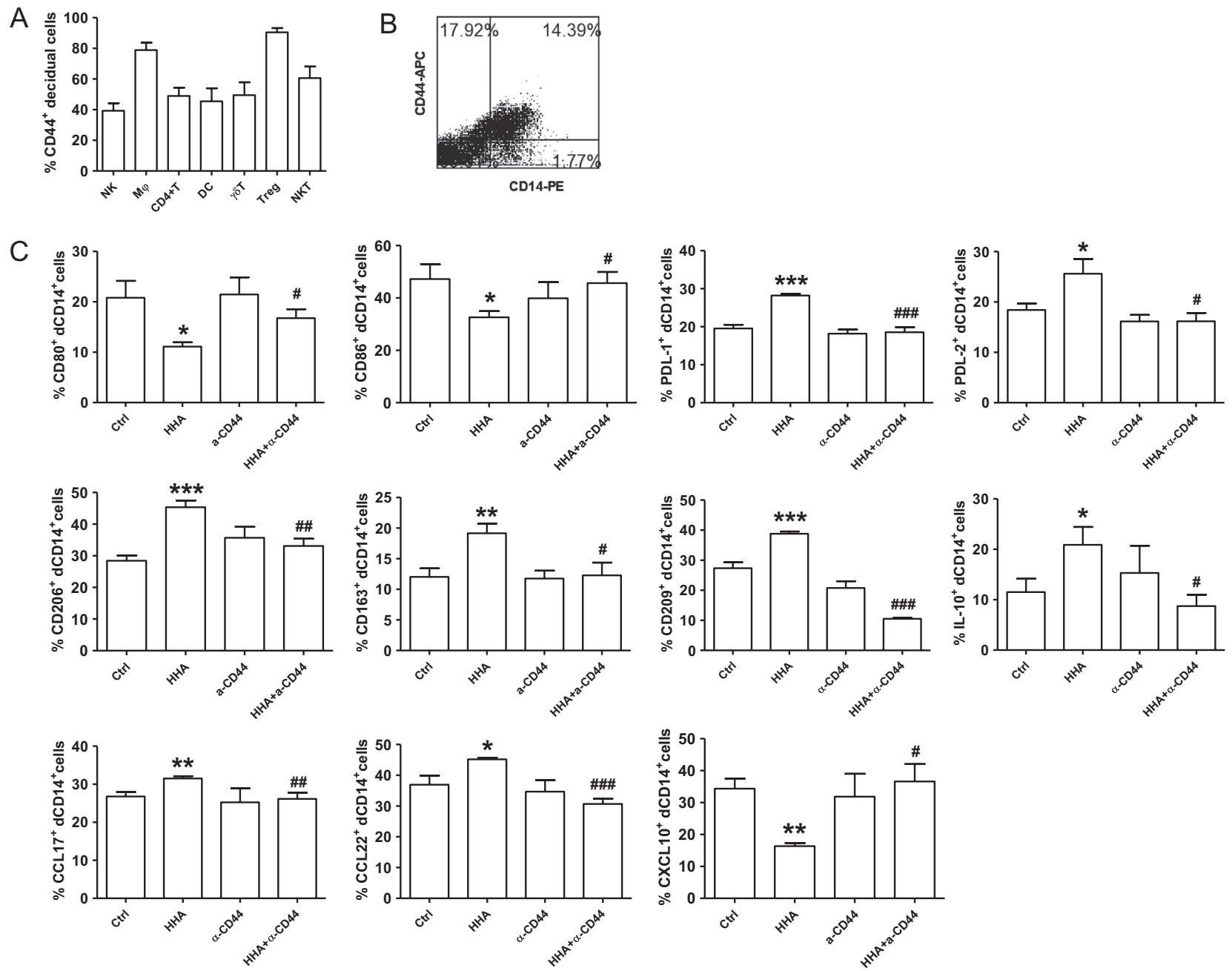

Figure 2 HMW-HA induces M2 polarization through CD44. (A) Quantitation of flow cytometric analysis of the expression of CD44 on DICs. (B) Flow cytometric analysis of the expression of CD44 on dM $\varphi s$. The flow cytometric plot is from one representative experiment among three separate experiments. (C) Quantitation of flow cytometric analysis of M1/M2 associated markers on dM $\varphi s$ stimulated with HMW-HA (HHA, $100 \mu \mathrm{g} / \mathrm{mL}$ ) in the presence or absence of anti-CD44 mAbs. Data are represented as the mean \pm S.E.M. for three separate experiments. $n=9 \mathrm{dM} \varphi \mathrm{s}$

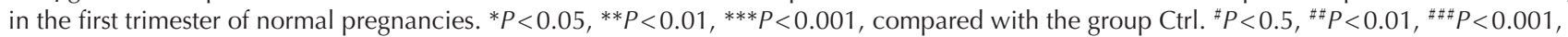
compared with the group HHA. 
not due to its cytotoxic effect, but that it resulted in the inhibition of the HMW-HA-mediated activity. We further confirmed that HMW-HA could activate the Akt signaling pathway in a time-dependent manner by Western blotting (Fig. 3B).

The activation of the signal transducer and activator of transcription 3 (STAT-3) and STAT-6 is essential for the expression of M2-associated markers and TAMs bioactivities (Sica \& Bronte 2007, Ishii et al. 2009). Our data showed that the phosphorylation levels of both STAT-3 and STAT-6 in dM 9 s were remarkably increased when treated with HMW-HA. Furthermore, the inhibition of PI3K suppressed the phosphorylation of STAT- 3 and STAT- 6 in dM $\varphi$ s stimulated by HMW-HA
(Fig. 3C). So, the PI3K/Akt-STAT-3/STAT-6 pathways were involved in the M2 polarization induced by HMW-HA.

\section{Tros instruct dM $\mathrm{M}$ s to an $\mathrm{M} 2$ phenotype via $H M W-H A$}

Previously, we have shown that Tros have the unique ability to instruct $T$ cells, and primary Tros could secrete HA continuously (Zhu et al. 2013b, Wang et al. 2015, 2016). Furthermore, in normal pregnancies, the HA produced by Tros is mostly HMW (data not shown). So we questioned whether Tros could also instruct macrophages and whether this effect was dependent on HA secretion. As expected, treatment
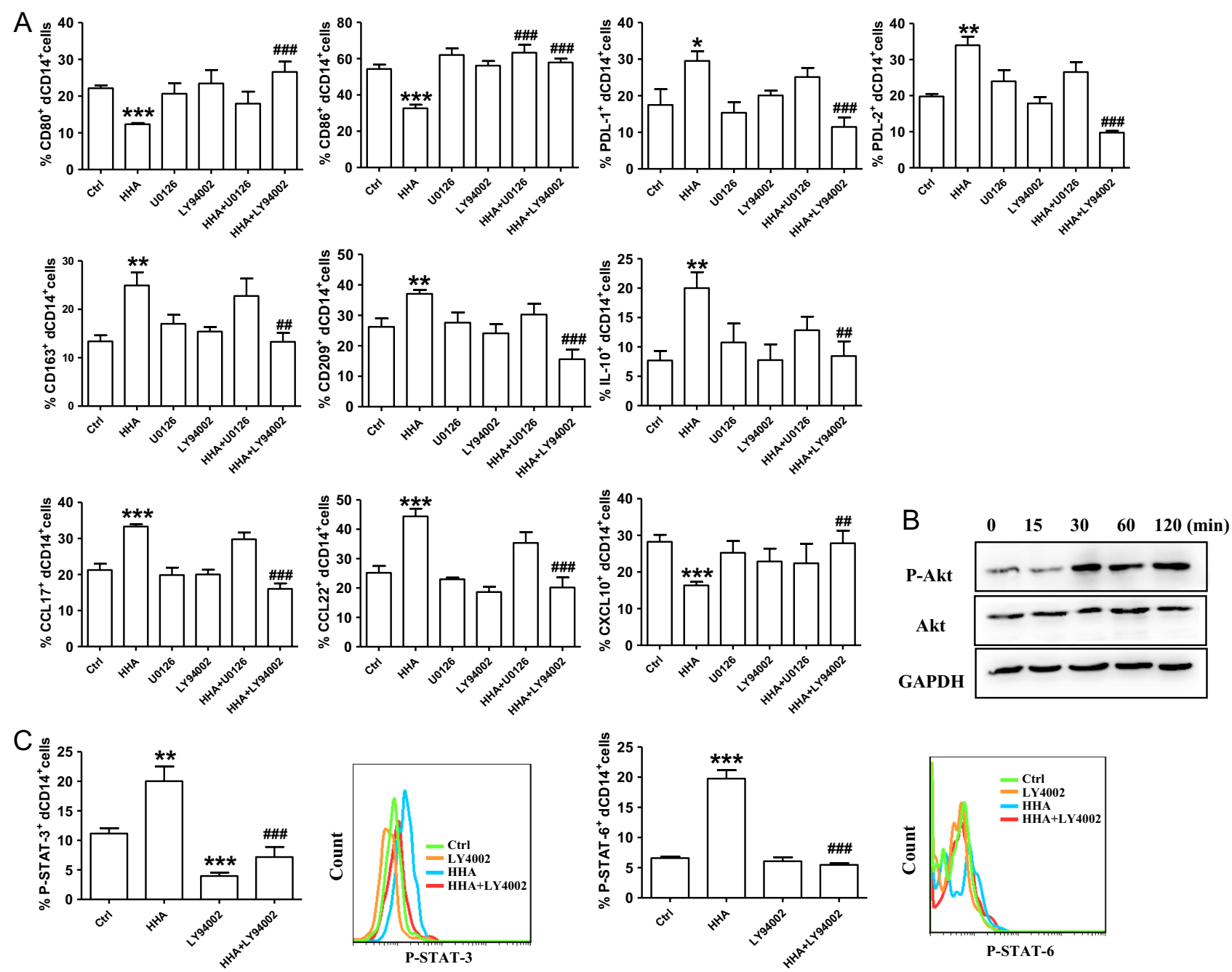

Figure 3 PI3K/Akt-STAT-3/STAT-6 pathways are involved in the M2 polarization. (A) Quantitation of flow cytometric analysis of M1/M2 associated markers on dM $\varphi$ s stimulated with $\mathrm{HMW}-\mathrm{HA}(\mathrm{HHA}, 100 \mu \mathrm{g} / \mathrm{mL})$ in the presence or absence of the indicated inhibitors of signal transduction. (B) dM $\varphi s$ were serum treated with $100 \mu \mathrm{g} / \mathrm{mL} \mathrm{HMW-HA}$ at a different time. A Western blot was used to analyze the total levels and phosphorylation levels of Akt. HMW-HA induced Akt phosphorylation in dM $\varphi s$ in a time-dependent manner. The image is representative of four individual experiments. (C) Flow cytometric analysis (right) and quantitation (left) of STAT-3 and STAT-6 phosphorylation in dM $\varphi$ s stimulated with HMW-HA $(\mathrm{HHA}, 100 \mu \mathrm{g} / \mathrm{mL})$ in the presence or absence of the PI3K signaling pathway inhibitor LY294002. The flow cytometric plot is from one representative experiment. Data are represented as the mean \pm S.E.M. for three separate experiments, $n=9$. ${ }^{*} P<0.05, * * P<0.01$, ${ }^{* * *} P<0.001$, compared with group Ctrl. ${ }^{\sharp \sharp} P<0.01,{ }^{\sharp \#} P<0.001$, compared with group HHA. 
of the TCM increased the expression of M2-associated markers, while decreasing the M1-associated markers of dM $\mathrm{s}$. Treatment with an HA antagonist peptide and/or blocking HA-CD44 interaction by anti-CD44neutralizing antibody significantly abrogated the Trosinduced M2 polarization, whereas CD80 and CD86 were upregulated (Fig. 4).

\section{Dysregulation of $d M \varphi s$ correlates with human miscarriages}

Our previous report has proven that lower HA secretion by Tros was observed in miscarriages than that of normal pregnancies (Zhu et al. 2013b). Because Tros could instruct dM $\varphi$ s into an M2 bias (Fig. 4), we analyzed the phenotype of $\mathrm{dM} \varphi \mathrm{s}$ from normal pregnant women and patients who experienced RSA to explore the possible clinical significance of dM $\varphi s$ instructed by Tros-secreted $\mathrm{HA}$. Flow cytometric analysis revealed a much lower expression of PDL-1, PDL-2, IL-10, IL-4, CD206, CD209 and CD163 on dMps from RSA compared with that from normal pregnancy (Fig. 5A, B and C). Conversely, the expression of CD80, CD86 and IL-12 by dM $\varphi$ S was much higher in RSA (Fig. $5 \mathrm{~A}$ and $\mathrm{B}$ ), whereas the expression of HLA-DR and ICOSL on $\mathrm{dM} \varphi$ s was not different between the two groups (Fig. 5A). Besides affecting the biological behaviors of Tros themselves (Zhu et al. 2013b), the reduced secretion of HA by Tros also affected the function of $\mathrm{dM} \varphi \mathrm{s}$, further leading to the development of RSA.

Next, we treated $\mathrm{dM} \varphi \mathrm{s}$ isolated from patients with RSA with $100 \mu \mathrm{g} / \mathrm{mL}$ HMW-HA to explore whether $\mathrm{HA}$ could rebalance the M1/M2 polarization of dM $\varphi s$ from RSA. As shown in Fig. 5D, $100 \mu \mathrm{g} / \mathrm{mL} \mathrm{HMW}-\mathrm{HA}$ upregulated the expression of CD206, CD209, CD163, PDL-1 and PDL-2 but downregulated the expression of CD80 and CD86 on dM 9 s from RSA. Furthermore, HMW-HA restored the production of IL-10 by dM $\varphi$ S isolated from patients with RSA, although it had no effect on the production of IL-4 and IL-12. These data indicated that the reduced secretion of HA may act as an early warning sign of adverse pregnancy, and HA may represent novel therapeutic strategies to prevent pregnancy loss.
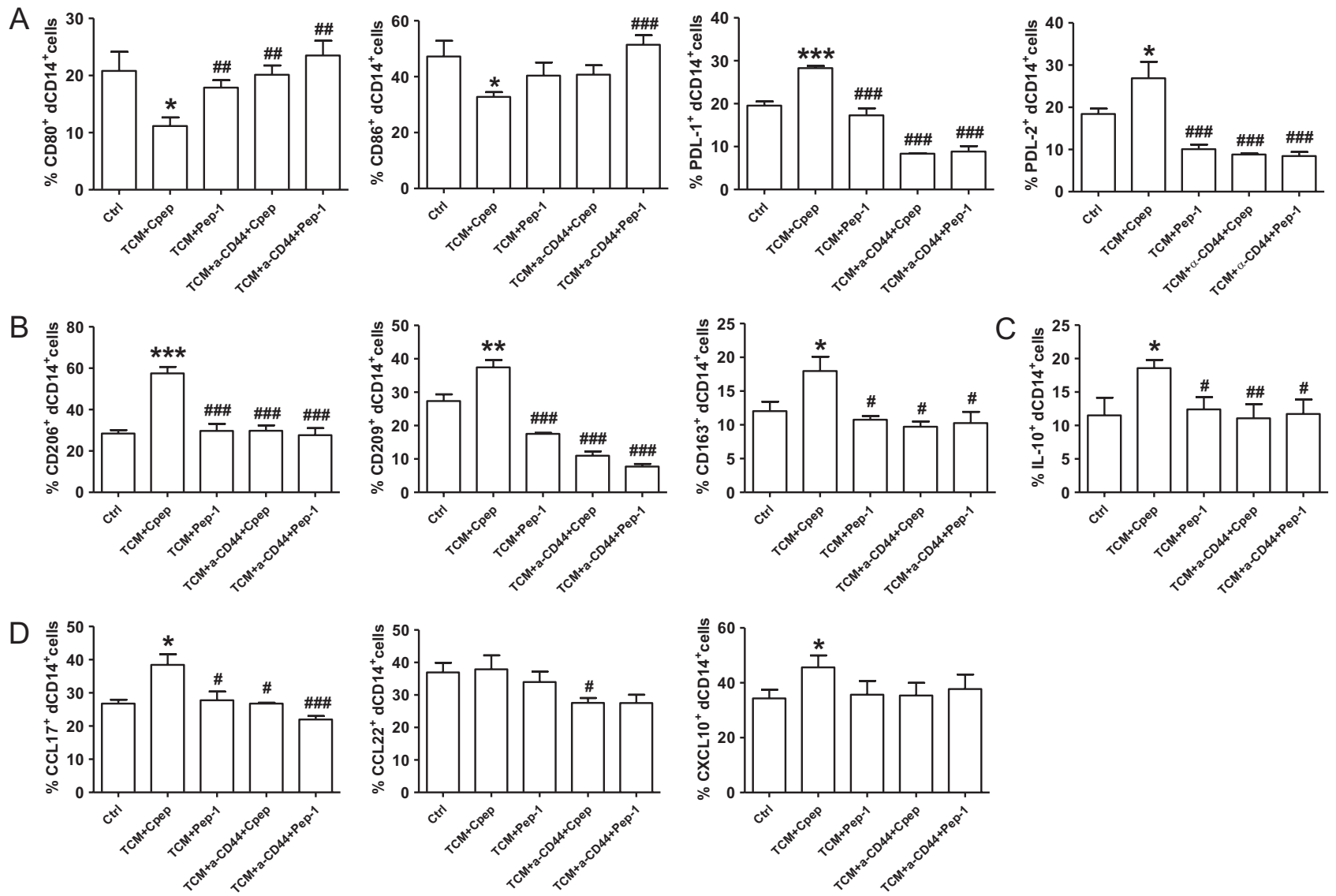

Figure 4 Tros instruct dM $\varphi$ s to an M2 phenotype via HMW-HA. Quantitation of flow cytometric analysis of M1/M2 associated markers on dM $\varphi$ s cultured with TCM in the presence or absence of pep-1 and Cpep with or without pretreatment of neutralizing anti-CD44 (20 $\mu \mathrm{g} / \mathrm{mL}) \mathrm{antibody}$. Data are represented as the mean \pm S.E.M. for three separate experiments, $n=12 .{ }^{*} P<0.05,{ }^{* *} P<0.01,{ }^{* * *} P<0.001$, compared with group Ctrl. ${ }^{\sharp} P<0.5,{ }^{\sharp} P<0.01,{ }^{\sharp \#} P<0.001$, compared with group TCM + Cpep. 

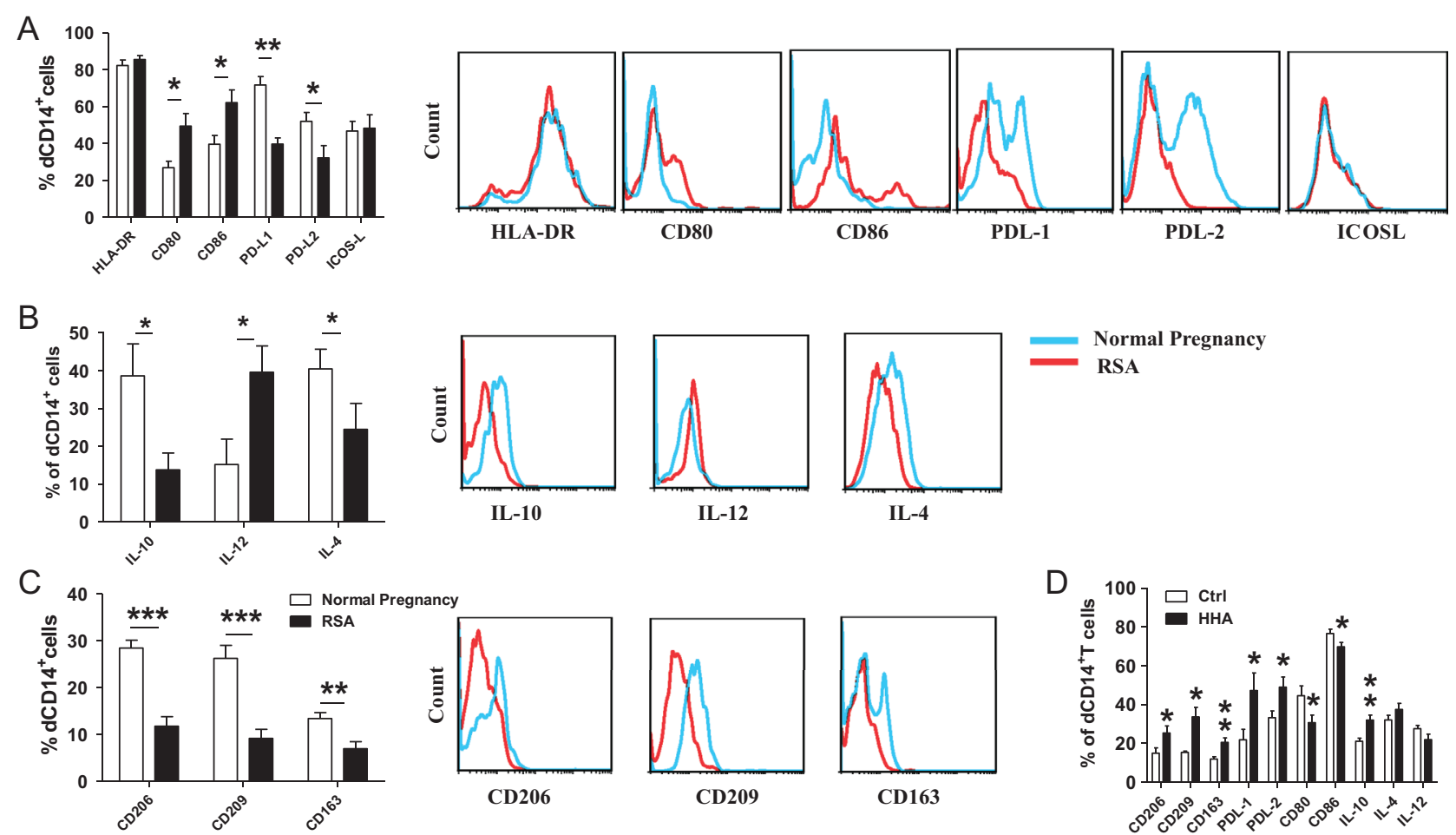

Figure 5 Dysregulation of $\mathrm{dM} \varphi \mathrm{s}$ correlates with human miscarriages. (A) Flow cytometric analysis (right) and quantitation (left) of MHC II and co-stimulatory molecules on dM $\varphi s$ from normal pregnancies $(n=12)$ and RSA $(n=19)$. (B) Flow cytometric analysis (right) and quantitation (left) of cytokine production by dM $\mathrm{d}$ s from normal pregnancies and RSA. (C) Flow cytometric analysis (right) and quantitation (left) of pattern recognition receptors on $\mathrm{dM} \varphi \mathrm{s}$ from normal pregnancy and RSA. The flow cytometric plot is from one representative experiment among six separate experiments. (D) Quantitation of flow cytometric analysis of the surface molecule expression and cytokine production by $\mathrm{dM} \varphi \mathrm{s}$ isolated from patients with RSA $(n=9)$ stimulated with HMW-HA $(\mathrm{HHA}, 100 \mu \mathrm{g} / \mathrm{mL})$ from three separate experiments. Data are represented as the mean \pm S.E.M. ${ }^{*} P<0.05,{ }^{* *} P<0.01,{ }^{* * *} P<0.001$.

\section{Discussion}

A successful pregnancy relies on adequate placental formation and the maternal immune system tolerating the semi-allogeneic fetus. Multiple mechanisms function to protect the fetus from attacks by the maternal immune system and promise the adequate invasion of extravillous Tros. In humans, dM $\varphi s$ display an M2 phenotype and may be involved in Tros invasion, vascular remodeling and the development of maternal-fetal tolerance (Petroff et al. 2002). Previously, we reported that a higher level and greater molecular mass of HA can promote Tros growth and invasion in an autocrine manner, which is beneficial to placentation, whereas the lower HA secretion by Tros was observed in miscarriages than in normal pregnancies (Zhu et al. 2013b). The current study shows that, during normal pregnancies in humans, Tros-derived HA induces M2 polarization by interacting with CD44 and activating the PI3K/AktSTAT3/STAT-6 pathway. This is further evidence of the delicate crosstalk between the fetus-derived Tros and maternal cells during normal pregnancies. Additionally, a decrease in $\mathrm{HA}$ secretion results in an abnormal function of macrophages, leading to RSA. Due to the fact that HA could restore the production of IL-10 and other normal pregnancy markers on $\mathrm{dM} \varphi \mathrm{s}$ isolated from patients with RSA, this indicates that HA may present novel therapeutic strategies to prevent pregnancy loss.

$\mathrm{HA}$ has received great attention as a versatile functional biopolymer since its discovery. The native form, HMW-HA, can be broken down into lower-molecularweight fragments in response to environmental cues, such as reactive oxygen species and $\mathrm{pH}$ (Stern et al. 2007). The molecular weight variants of HA have been used in a variety of biomedical applications eliciting varying biologic responses (Highley et al. 2016). For example, LMW-HA was identified as inducing potent pro-inflammatory responses (Powell \& Horton 2005), whereas HMW-HA was reported to regulate osteoclast formation (Ariyoshi et al. 2014) and create a specific microenvironment favorable for tumor development (Chanmee et al. 2016). Because of the lack of a standard size for HMM- and LMM-HA, HA may serve various roles in different studies. We found that HMW-HA could induce $\mathrm{M} 2$ polarization of $\mathrm{dM} \varphi \mathrm{s}$, but $\mathrm{MMW}$ - and LMW-HA had no effects on the expression of biomarkers of M1/M2 (Fig. 1). However, it has been shown in a study that macrophages correspond to pro-inflammatory responses for LMW-HA (Rayahin et al. 2015). This may due to the different molecular weights of HA used 


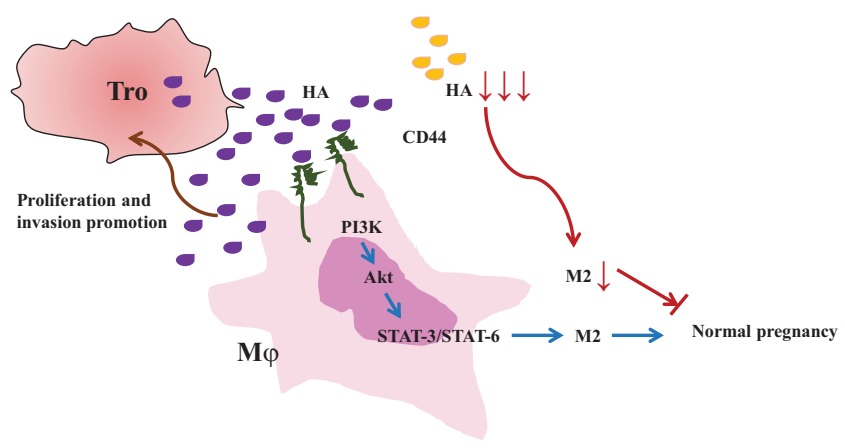

Figure $6 \mathrm{~A}$ schematic diagram of Tros-derived HA induced M2 polarization at the maternal-fetal interface. Tros secrete HA, which in turn promotes the invasion and proliferation of human Tros. Tros-derived $\mathrm{HMW}-\mathrm{HA}$ instructs dM $\varphi s$ to transform into immunosuppressive M2 through interacting with CD44 and activating the downstream PI3K/Akt-STAT-3/STAT-6 signaling pathways. While in RSA, the secretion of HA by Tros was decreased, and the M2 bias was disrupted at the maternal-fetal interface, which was harmful to the maintenance of normal pregnancy.

$(\leq 5 \mathrm{kDa}$ in the report) and the different characteristics between $\mathrm{d} M \varphi s$ and murine macrophages.

Our results also showed that co-culture of Tros increased the expression of $\mathrm{M} 2$-associated markers while decreasing the $\mathrm{M} 1$-associated markers of dM $\varphi$ s. With the higher expression of IL-10 and CD163, CD206, CD209, PDL-1 and PDL-2, dM 9 s could better exert immunosuppressive effects and remove pathogens, thus promoting immune tolerance at the maternal-fetal interface (Smith et al. 2004, Terrazas et al. 2005, Tang et al. 2015). Treatment with an HA antagonist peptide significantly abrogated the Tros-induced M2 polarization. This is also supported by a recent study that displayed a link between HA accumulation and large numbers of M2-like macrophages (Tiainen et al. 2015). Our results provide further evidence that Tros could educate maternal immune cells to develop a unique phenotype and tolerate the fetus.

CD44 is the major cell-surface receptor for $\mathrm{HA}$, and HA-CD44 signaling appears to be significant in regulating immunological responses, such as T cells recruitment, mature $\mathrm{B}$ cells activation and monocytes deactivation (Jiang et al. 2011). We found that antagonizing the HACD44 interaction on $\mathrm{d} M \varphi s$, or between Tros and $\mathrm{dM} \varphi \mathrm{s}$, significantly inhibits the phenotype transformation, suggesting that the induction of $\mathrm{M} 2$ by $\mathrm{HA}$ is triggered by binding to CD44. However, besides CD44, HA could also bind to other receptors. For example, it has been reported in a study that CD44 and TLR4 were both engaged in the HA-mediated deactivation of monocytes (Del et al. 2005). Our study cannot exclude other HA receptors in M2 conversion at the maternal-fetal interface, which needs further investigation.

The signaling pathways in $\mathrm{dM} \varphi \mathrm{s}$ polarization are mostly unclear. It is well documented that ERK1/2 and
PI3K/Akt signal cascades are closely connected to the function of HA (Zhu et al. 2013a,b). Our data showed that the PI3K/Akt signal activated by HA-CD44 interaction induced the phenotype transformation of $\mathrm{dM} \varphi \mathrm{s}$, implying a novel role of the PI3K/Akt pathway in macrophages function. Of note, it was found in one study that the PI3K/Akt pathway could also inhibit abnormal macrophage activation in the placenta (Zhou et al. 2017). STAT-3 and STAT-6 signals in macrophages are involved in the regulation of immune responses and are master regulator of $\mathrm{M} 2$ genes (Welch et al. 2002, Ishii et al. 2009). The activation of STAT-3 and STAT-6 is inhibited by suppressing the PI3K/AKT signal in dM $\varphi s$. Our data suggest that the PI3K/Akt-STAT-3/STAT-6 pathway is the molecular cue responsible for HA-mediated M2 formation at the maternal-fetal interface.

Abnormally activated $\mathrm{d} M \varphi \mathrm{s}$ can disrupt the tolerance at the maternal-fetal interface, which is closely associated with RSA, pre-eclampsia and premature delivery (Xu et al. 2016). Our data, together with previous studies indicating M1 dominance in miscarriages (Shimada et al. 2018, Tsao et al. 2018), suggest that $\mathrm{M} 1$ bias is detrimental to pregnancy. HA was able to induce $\mathrm{M} 2$ polarization at the maternalfetal interface, and the secretion of HA by Tros from RSA significantly decreased (Zhu et al. 2013b). Furthermore, HA could restore the M2 phenotype of $\mathrm{dM} \varphi s$ isolated from patients with RSA. Based on these results, we conclude that failure to maintain M2 bias resulting from the low $\mathrm{HA}$ microenvironment at the maternal-fetal interface may be one of the major reasons leading to RSA. However, although Trosderived HA polarizes M2 in vitro, its precise role in vivo remains to be established.

In summary, we have demonstrated that a high level and mature state of $\mathrm{HA}$ is beneficial to the maintenance of normal pregnancy. Additionally, Tros-derived HA instructs $\mathrm{dM} \varphi \mathrm{s}$ to transform into immunosuppressive M2 through interacting with CD44 and activating the downstream PI3K/Akt-STAT-3/STAT-6 signaling pathways (Fig. 6). Our study provides new insights into the mechanisms underlying the formation of $\mathrm{M} 2$ bias during normal pregnancies, which may be helpful in pregnancy loss immunotherapy.

\section{Supplementary data}

This is linked to the online version of the paper at https://doi.org/10.1530/REP-18-0450.

\section{Declaration of interest}

The authors declare that there is no conflict of interest that could be perceived as prejudicing the impartiality of the research reported. 


\section{Funding}

This work was supported by grant from the Nature Science Foundation from National Nature Science Foundation of China (NSFC) (31700799 to S C W, 81630036, 91542116 and 31570920 to M R D, 81490744 to DJL, 81501334 to Y T, 81401273 to R Z), the Youth Science Fund Project of the Natural Science Foundation of Jiangsu Province (BK20140276 to R Z), Jiangsu Key Youth Medical Talents (QNRC2016244 to R Z), the Introduction Project of Suzhou Clinical Medicine Expert Team (SZYJTD201708 to R Z, H L and D J L), the Shanghai Sailing Program (17YF1411600 to S CW), Suzhou Key Medical Center (SZZX201505 to R Z and H L), the National Basic Research Program of China (2015CB943300 to D J L and M R D), and National Key R\&D Program of China (2017YFC1001403 to D J L and M R D), Training Program for Young Talents of Shanghai Health System (2018YQ07 to S C W), Development Fund of Shanghai Talents (2018110 to S C W), the Innovation-oriented Science and Technology Grant from NHC Key Laboratory of Reproduction Regulation (CX2017-2 to MRD and DJL), the key talents of Gusu Health to R Z and the cultivate talents of Jiangsu 333 project to R Z.

\section{References}

Akgul Y, Word RA, Ensign LM, Yamaguchi Y, Lydon J, Hanes J \& Mahendroo M 2014 Hyaluronan in cervical epithelia protects against infection-mediated preterm birth. Journalof Clinical Investigation 124 5481-5489. (https://doi.org/10.1172/JCl78765)

Arck PC \& Hecher K 2013 Fetomaternal immune cross-talk and its consequences for maternal and offspring's health. Nature Medicine 19 548-556. (https://doi.org/10.1038/nm.3160)

Ariyoshi W, Okinaga T, Knudson CB, Knudson W \& Nishihara T 2014 High molecular weight hyaluronic acid regulates osteoclast formation by inhibiting receptor activator of NF-kappaB ligand through Rho kinase. Osteoarthritis Cartilage 22 111-120. (https://doi.org/10.1016/j. joca.2013.10.013)

Care AS, Diener KR, Jasper MJ, Brown HM, Ingman WV \& Robertson SA 2013 Macrophages regulate corpus luteum development during embryo implantation in mice. Journal of Clinical Investigation 123 3472-3487. (https://doi.org/10.1172/JCl60561)

Chanmee T, Ontong P \& Itano N 2016 Hyaluronan: a modulator of the tumor microenvironment. Cancer Letters 375 20-30. (https://doi. org/10.1016/j.canlet.2016.02.031)

Del FC, Otero K, Gomez-Garcia L, Gonzalez-Leon MC, SolerRanger L, Fuentes-Prior P, Escoll P, Baos R, Caveda L, Garcia F et al. 2005 Tumor cells deactivate human monocytes by up-regulating IL-1 receptor associated kinase-M expression via CD44 and TLR4. Journal of Immunology 174 3032-3040. (https://doi.org/10.4049/ jimmunol.174.5.3032)

Dicker KT, Gurski LA, Pradhan-Bhatt S, Witt RL, Farach-Carson MC \& Jia, X 2014 Hyaluronan: a simple polysaccharide with diverse biological functions. Acta Biomaterialia 10 1558-1570. (https://doi.org/10.1016/j. actbio.2013.12.019)

Egashira $M$, Hirota $Y$, Shimizu-Hirota R, Saito-Fujita T, Haraguchi $H$, Matsumoto L, Matsuo M, Hiraoka T, Tanaka T, Akaeda S et al. 2017 $\mathrm{F} 4 / 80$ + macrophages contribute to clearance of senescent cells in the mouse postpartum uterus. Endocrinology 158 2344-2353. (https://doi. org/10.1210/en.2016-1886)

Heldin P, Karousou E, Bernert B, Porsch H, Nishitsuka K, \& Skandalis SS 2008 Importance of hyaluronan-CD44 interactions in inflammation and tumorigenesis. Connective Tissue Research 49 215-218. (https://doi. org/10.1080/03008200802143323)

Highley CB, Prestwich GD \& Burdick JA 2016 Recent advances in hyaluronic acid hydrogels for biomedical applications. Current Opinion in Biotechnology 40 35-40. (https://doi.org/10.1016/j. copbio.2016.02.008)
Ishii M, Wen H, Corsa CA, Liu T, Coelho AL, Allen RM, Carson WT, Cavassani KA, Li X, Lukacs NW et al. 2009 Epigenetic regulation of the alternatively activated macrophage phenotype. Blood 114 3244-3254. (https://doi.org/10.1182/blood-2009-04-217620)

Jaiswal MK, Mallers TM, Larsen B, Kwak-Kim J, Chaouat G, GilmanSachs A, \& Beaman KD 2012 V-ATPase upregulation during early pregnancy: a possible link to establishment of an inflammatory response during preimplantation period of pregnancy. Reproduction 143 713-725. (https://doi.org/10.1530/REP-12-0036)

Jiang D, Liang J, Fan J, Yu S, Chen S, Luo Y, Prestwich GD, Mascarenhas MM, Garg HG, Quinn DA et al. 2005 Regulation of lung injury and repair by Toll-like receptors and hyaluronan. Nature Medicine 11 1173-1179. (https://doi.org/10.1038/nm1315)

Jiang D, Liang J, \& Noble PW 2011 Hyaluronan as an immune regulator in human diseases. Physiological Reviews 91 221-264. (https://doi. org/10.1152/physrev.00052.2009)

Lash GE, Pitman H, Morgan HL, Innes BA, Agwu CN \& Bulmer JN 2016 Decidual macrophages: key regulators of vascular remodeling in human pregnancy. Journal of Leukocyte Biology 100 315-325. (https://doi. org/10.1189/jlb.1A0815-351R)

Mills CD, Kincaid K, Alt JM, Heilman MJ \& Hill AM 2000 M-1/M-2 macrophages and the Th1/Th2 paradigm. Journal of Immunology $\mathbf{1 6 4}$ 6166-6173. (https://doi.org/10.4049/jimmunol.164.12.6166)

Murray PJ, Allen JE, Biswas SK, Fisher EA, Gilroy DW, Goerdt S, Gordon S, Hamilton JA, Ivashkiv LB \& Lawrence T 2014 Macrophage activation and polarization: nomenclature and experimental guidelines. Immunity 41 14-20. (https://doi.org/10.1016/j.immuni. 2014.06.008)

Petroff MG, Sedlmayr P, Azzola D \& Hunt JS 2002 Decidual macrophages are potentially susceptible to inhibition by class la and class Ib HLA molecules. Journal of Reproductive Immunology 56 3-17. (https://doi. org/10.1016/S0165-0378(02)00024-4)

Powell JD \& Horton MR 2005 Threat matrix: low-molecular-weight hyaluronan (HA) as a danger signal. Immunologic Research 31 207-218. (https://doi.org/10.1385/IR:31:3:207)

Rayahin JE, Buhrman JS, Zhang Y, Koh TJ \& Gemeinhart RA 2015 High and low molecular weight hyaluronic acid differentially influence macrophage activation. ACS Biomaterials Science and Engineering 1 481-493. (https://doi.org/10.1021/acsbiomaterials.5b00181)

Shimada S, Ebina Y, lijima N, Deguchi M \& Yamada H 2018 Decidual CD68(+) HLA-DR(+) CD163(-) M1 macrophages increase in miscarriages with normal fetal chromosome. American Journal of Reprodective Immunology 79 e12791. (https://doi.org/10.1111/aji.12791)

Sica A, \& Bronte V 2007 Altered macrophage differentiation and immune dysfunction in tumor development. Journal of Clinical Investigation $\mathbf{1 1 7}$ 1155-1166. (https://doi.org/10.1172/JCI31422)

Smith P, Walsh CM, Mangan NE, Fallon RE, Sayers JR, McKenzie AN \& Fallon PG 2004 Schistosoma mansoni worms induce anergy of T cells via selective up-regulation of programmed death ligand 1 on macrophages. Journal of Immunology 173 1240-1248. (https://doi.org/10.4049/ jimmunol.173.2.1240)

Stern R, Kogan G, Jedrzejas MJ \& Soltes L 2007 The many ways to cleave hyaluronan. Biotechnology Advances 25 537-557. (https://doi. org/10.1016/j.biotechadv.2007.07.001)

Svensson-Arvelund J \& Ernerudh J 2015 The role of macrophages in promoting and maintaining homeostasis at the fetal-maternal interface. American Journal of Reproductive Immunology 74 100-109. (https://doi. org/10.1111/aji.12357)

Tang MX, Hu XH, Liu ZZ, Kwak-Kim J \& Liao AH 2015 What are the roles of macrophages and monocytes in human pregnancy? Journal of Reproductive Immunology 112 73-80. (https://doi.org/10.1016/j. jri.2015.08.001)

Terrazas LI, Montero D, Terrazas CA, Reyes JL \& Rodriguez-Sosa M 2005 Role of the programmed death- 1 pathway in the suppressive activity of alternatively activated macrophages in experimental cysticercosis. International Journal for Parasitology 35 1349-1358. (https://doi. org/10.1016/j.ijpara.2005.06.003)

Tiainen S, Tumelius R, Rilla K, Hamalainen K, Tammi M, Tammi R, Kosma VM, Oikari S \& Auvinen P 2015 High numbers of macrophages, especially M2-like (CD163-positive), correlate with hyaluronan accumulation and poor outcome in breast cancer. Histopathology $\mathbf{6 6}$ 873-883. (https://doi.org/10.1111/his.12607) 
Tsao FY, Wu MY, Chang YL, Wu CT \& Ho HN 2018 M1 macrophages decrease in the deciduae from normal pregnancies but not from spontaneous abortions or unexplained recurrent spontaneous abortions. Journal of the Formosan Medical Association 117 204-211. (https://doi. org/10.1016/j.jfma.2017.03.011)

Wang SC, Li YH, Piao HL, HongXW, Zhang D, Xu YY, Tao Y, Wang Y, Yuan MM, Li DJ et al. 2015 PD-1 and Tim-3 pathways are associated with regulatory $\mathrm{CD} 8+\mathrm{T}$-cell function in decidua and maintenance of normal pregnancy. Cell Death and Disease 6 e1738. (https://doi. org/10.1038/cddis.2015.112)

Wang S, Zhu X, Xu Y, Zhang D, Li Y, Tao Y, Piao H, Li D \& Du M 2016 Programmed cell death-1 (PD-1) and T-cell immunoglobulin mucin-3 (Tim-3) regulate CD4+ T cells to induce Type 2 helper T cell (Th2) bias at the maternal-fetal interface. Human Reproduction 31 700-711. (https:// doi.org/10.1093/humrep/dew019)

Welch JS, Escoubet-Lozach L, Sykes DB, Liddiard K, Greaves DR \& Glass CK 2002 TH2 cytokines and allergic challenge induce Ym1 expression in macrophages by a STAT6-dependent mechanism. Journal of Biological Chemistry 277 42821-42829. (https://doi.org/10.1074/jbc. M205873200)

Xu Y, Romero R, Miller D, Kadam L, Mial TN, Plazyo O, Garcia-Flores V, Hassan SS, Xu Z, Tarca AL et al. 2016 An M1-like macrophage polarization in decidual tissue during spontaneous preterm labor that is attenuated by rosiglitazone treatment. Journal of Immunology 196 2476-2491. (https://doi.org/10.4049/jimmunol.1502055)

Zhang G, Guo L, Yang C, Liu Y, He Y, Du Y, Wang W \& Gao F 2016 A novel role of breast cancer-derived hyaluronan on inducement of
M2-like tumor-associated macrophages formation. Oncolmmunology 5 e1172154. (https://doi.org/10.1080/2162402X.2016.1172154)

Zhang YH, He M, Wang Y \& Liao AH 2017 Modulators of the balance between $\mathrm{M} 1$ and $\mathrm{M} 2$ macrophages during pregnancy. Frontiers in Immunology 8 120. (https://doi.org/10.3389/fimmu.2017.00120)

Zhou J, Miao H, Li X, Hu Y, Sun H \& Hou Y 2017 Curcumin inhibits placental inflammation to ameliorate LPS-induced adverse pregnancy outcomes in mice via upregulation of phosphorylated Akt. Inflammation Research 66 177-185. (https://doi.org/10.1007/s00011-016-1004-4)

Zhu R, Huang YH, Tao Y, Wang SC, Sun C, Piao HL, Wang XQ, Du MR \& Li DJ 2013a Hyaluronan up-regulates growth and invasion of trophoblasts in an autocrine manner via PI3K/AKT and MAPK/ERK1/2 pathways in early human pregnancy. Placenta 34 784-791. (https://doi. org/10.1016/j.placenta.2013.05.009)

Zhu R, Wang SC, Sun C, Tao Y, Piao HL, Wang XQ, Du MR \& Li DJ 2013 b Hyaluronan-CD44 interaction promotes growth of decidual stromal cells in human first-trimester pregnancy. PLOS ONE 8 e74812. (https:// doi.org/10.1371/journal.pone.0074812)

Received 24 August 2018

First decision 26 September 2018

Revised manuscript received 19 November 2018

Accepted 6 December 2018 